\title{
Brownian Motion in Gravitationally Interacting Systems
}

\section{Citation}

Chatterjee, Pinaki, Lars Hernquist, and Abraham Loeb. 2002. "Brownian Motion in Gravitationally Interacting Systems." Physical Review Letters 88 (12). https://doi.org/10.1103/ physrevlett.88.121103.

\section{Permanent link}

http://nrs.harvard.edu/urn-3:HUL.InstRepos:41381791

\section{Terms of Use}

This article was downloaded from Harvard University's DASH repository, and is made available under the terms and conditions applicable to Other Posted Material, as set forth at http:// nrs.harvard.edu/urn-3:HUL.InstRepos:dash.current.terms-of-use\#LAA

\section{Share Your Story}

The Harvard community has made this article openly available.

Please share how this access benefits you. Submit a story.

Accessibility 


\title{
Brownian Motion in Gravitationally-Interacting Systems
}

\author{
Pinaki Chatterjee, Lars Hernquist \& Abraham Loeb \\ Harvard-Smithsonian Center for Astrophysics, 60 Garden Street, Cambridge, MA 02138, USA \\ pchatterjee@cfa.harvard.edu,lars@cfa.harvard.edu, aloeb@cfa.harvard.edu
}

(October 29, 2018)

\begin{abstract}
We derive a model that describes the motion of a Brownian particle in a system which is dominated by gravitational forces. An example of such a system is a massive black hole immersed in a cluster of stars. We compute the dispersion in the position and velocity of such a black hole, and examine whether it achieves a state of equipartition of kinetic energy with the stars. This problem has been considered before only for stellar systems with an isothermal Maxwellian distribution of velocities; here we study other examples and confirm our calculations with N-body simulations. In certain cases, depending on the stellar distribution function, the black hole can acquire a steady state kinetic energy which is very far from equipartition relative to the stars.

PACS numbers: 95.10.Ce, 45.50.Pk, 05.40.Jc, 98.10.+z, 98.62.Js
\end{abstract}

Brownian motion in stellar systems was studied by Chandrasekhar [1], who recognized that the force acting on an object in a stellar system consists of two independent contributions: one part, which originates from the "smoothed-out" average distribution of matter in the stellar system, varies slowly with position and time; the second part, which arises from discrete encounters with individual stars, fluctuates much more rapidly. The smooth force itself consists of the restoring force arising from the potential of the aggregate distribution of stars, and the dissipative force of dynamical friction which causes the object to decelerate as it moves through the stellar background [2]. This is similar to the Langevin model of Brownian motion, which describes the irregular motions of dust grains immersed in a gas of light molecules: the Brownian particle experiences a frictional force proportional to its velocity, and a random, rapidly fluctuating force owing to the large rate of collisions it suffers with the gas molecules in its neighborhood.

In a stellar system, the analog of the gas molecules is the stars, and the Brownian particle corresponds to any object which is much more massive than the stars and moves much more slowly than they do. An astrophysically relevant example is a massive black hole at the center of a dense stellar system such as a globular cluster or the nucleus of a galaxy. We would like to extend the Langevin method of analysis to such a problem.

Consider, therefore, a black hole of mass $m$ in a cluster of stars of total mass $M$ which we take to be described by the spherically symmetric density and potential profiles $\rho(r)$ and $\Phi(r)$, respectively, where $\mathbf{r}$ is the radial position vector from the center of the stellar system, which is taken as the origin. The phase space distribution function, which in general will depend both on position $\mathbf{r}$, and velocity $\mathbf{v}$, is defined such that $f(\mathbf{r}, \mathbf{v}) d^{3} \mathbf{r} d^{3} \mathbf{v}$ is the mass in stars in the phase space volume $d^{3} \mathbf{r} d^{3} \mathbf{v}$. We assume that in a spherically symmetric stellar model, $f$ is a function only of the energy per unit mass $E$, where $E=\frac{1}{2} v^{2}+\Phi(r)$.

A black hole in this stellar system is subject to three forces. The first is the restoring force of the stellar potential, $\mathbf{F}=-m \nabla \Phi(r)$, where $\mathbf{r}$ is the position vector of the black hole. For a stellar system with a non-cuspy core, this reduces to the form $\mathbf{F}=-k \mathbf{r}$, where $k$ is independent of $r$ for small values of $r$.

The second force on the black hole is the dissipative force of dynamical friction which causes it to decelerate as it moves through the stellar background. We use for this the well-known Chandrasekhar formula ( [2], [3]) $\mathbf{F}=$ $-\beta \mathbf{v}$, where

$$
\beta=16 \pi^{2} \ln \Lambda G^{2} m\left(m+m_{\star}\right) \frac{\int_{0}^{v} f(r, u) u^{2} d u}{v^{3}} .
$$

In the above, $\mathbf{v}$ is the velocity of the black hole, $m_{\star}$ is the mass of each star (in the following, we take all stars to have equal masses, for simplicity), and $\ln \Lambda$ is the "Coulomb logarithm" [3]. Since the black hole remains close to the origin and moves very slowly compared with the stars, we may replace $f(r, u)$ in the integral by $f(r, 0)$, and evaluate it at $r \rightarrow 0$ to obtain [3]:

$$
\beta \rightarrow\left(16 \pi^{2} / 3\right) \ln \Lambda G^{2} m\left(m+m_{\star}\right) f(0,0) .
$$

The third force on the black hole fluctuates on a timescale which is extremely short compared to the above two forces 沺, and arises from random discrete encounters between the black hole and the stars. Denoting this stochastic force as $\mathbf{F}(t)$, we can write the equation of motion of the black hole as

$$
m \ddot{\mathbf{r}}(t)+\beta \dot{\mathbf{r}}(t)+k \mathbf{r}(t)=\mathbf{F}(t),
$$

which is the equation of motion of a harmonically bound Brownian particle. 
The spatial components of this linear vector equation are separable into equivalent components:

$$
m \ddot{x}(t)+\beta \dot{x}(t)+k x(t)=F_{x}(t) .
$$

The stochastic force is defined only statistically. Since it is random and rapidly varying, we expect it to be zero on average and uncorrelated with itself at different times:

$$
\left\langle F_{x}(t)\right\rangle=0, \quad\left\langle F_{x}\left(t_{1}\right) F_{x}\left(t_{2}\right)\right\rangle=C \delta\left(t_{1}-t_{2}\right),
$$

where $\delta$ is the Dirac delta function and the angular brackets denote an average over an ensemble of "similarly prepared" systems of stars in each of which the black hole has the same initial position and velocity. We will later show how to determine the value of $C$.

We have shown in detail in Ref. [4 how Eqs. (4) and (5) can be combined with the Fokker-Planck equation to derive the probability distributions of the black hole's position and velocity in the stationary state (i.e., when initial transients - which decay exponentially fast - die out and these distributions become time-independent); these turn out to be independent Gaussian distributions:

$$
\begin{gathered}
W(x)=\sqrt{2 \gamma / \pi C} \omega_{0} m \exp \left[-(2 \gamma / C) \omega_{0}^{2} m^{2} x^{2}\right], \\
W\left(v_{x}\right)=\sqrt{2 \gamma / \pi C} m \exp \left[-(2 \gamma / C) m^{2} v_{x}^{2}\right],
\end{gathered}
$$

where $\gamma=\beta / 2 m$ and $\omega_{0}=\sqrt{k / m}$. Note that $v_{x} \equiv \dot{x}$.

It was shown in [3] (their Eq. [8-66]) that the rate of change of kinetic energy of the black hole is given by

$$
\begin{aligned}
d\left(m v^{2} / 2\right) / d t= & 16 \pi^{2} \ln \Lambda G^{2} m m_{\star}\left[\int_{v}^{\infty} f(r, u) u d u\right. \\
& \left.-m /\left(m_{\star} v\right) \int_{0}^{v} f(r, u) u^{2} d u\right] .
\end{aligned}
$$

The first term describes the "heating" of the black hole by fluctuations in the stellar system, and the second term describes the "cooling" or dissipative effect of dynamical friction. Averaged over the ensemble in the stationary state, the two terms above should sum to zero. Again, setting $f(r, u)$ in integrals of the form $\int_{0}^{v} \ldots f(r, u) d u$ to $f(r, 0)$, we can derive

$$
\left\langle v^{2}\right\rangle=\left(3 m_{\star} / m\right) \int_{0}^{\infty} f(r, u) u d u / f(r, 0)=3\left\langle v_{x}^{2}\right\rangle,
$$

since the three velocity components are equivalent and independent of each other. From Eqs. (6) and (7),

$$
\left\langle x^{2}\right\rangle=C / 4 \gamma m^{2} \omega_{0}^{2}, \quad\left\langle v_{x}^{2}\right\rangle=C / 4 \gamma m^{2} .
$$

Combining Eqs. (9) and (10), we obtain the following expressions for $\left\langle x^{2}\right\rangle$ and $C$ :

$$
\left\langle x^{2}\right\rangle=\left(m_{\star} / m \omega_{0}^{2}\right) \int_{0}^{\infty} f(r, u) u d u / f(r, 0),
$$

$$
C=4 \gamma m m_{\star} \int_{0}^{\infty} f(r, u) u d u / f(r, 0) .
$$

We wish to have a measure for how far the black hole is from equipartition of kinetic energy between itself and the stars surrounding it in the core of the stellar system. Since the square of the stellar velocity dispersion is

$$
\overline{v_{\star}^{2}}=\int_{0}^{\infty} u^{2} f(r, u) 4 \pi u^{2} d u / \int_{0}^{\infty} f(r, u) 4 \pi u^{2} d u,
$$

we obtain by using Eq. (9):

$$
\left\langle v^{2}\right\rangle / \overline{v_{\star}^{2}}=\eta m_{\star} / m,
$$

where

$$
\eta=\frac{3 \int_{0}^{\infty} f(r, u) u d u \quad \int_{0}^{\infty} f(r, u) u^{2} d u}{f(r, 0) \int_{0}^{\infty} f(r, u) u^{4} d u}
$$

If $\eta$ is evaluated in the limit $r \rightarrow 0$, it measures the deviation of the black hole from equipartition of kinetic energy between it and the stars in the core surrounding it. When $\eta=1$, there is exact equipartition. We can take various stellar models and examine what the black hole's stationary state dynamics would be in each case.

Maxwellian distribution. Suppose the phase space distribution of stars to be given by a Maxwellian distribution with velocity dispersion $\sigma$ :

$$
f(r, u) \propto \exp \left(-u^{2} / 2 \sigma^{2}\right)
$$

A simple calculation then gives $\eta=1$. This is not surprising: the analogy in this case is with a Brownian particle immersed in a gas of molecules with a well-defined temperature [3]; in the steady state, such a Brownian particle will be in exact equipartition with the molecules.

The King model. The simplest model for which the distribution function is a pure Maxwellian is the "isothermal sphere", which is not a very physical description of actual stellar systems since it has infinite total mass. A commonly used alternative that resembles the isothermal sphere at small radii (where stars have large absolute values of energy per unit mass) is the King model (see [5], [3):

$$
f(E)= \begin{cases}\rho_{1}\left(2 \pi \sigma^{2}\right)^{-\frac{3}{2}}\left(e^{-E / \sigma^{2}}-1\right) & \text { if } E<0 \\ 0 & \text { if } E \geq 0,\end{cases}
$$

where $\rho_{1}$ is independent of $E$. In Eq. (15), the upper velocity limit of the integrals is now $\sqrt{2 \sigma^{2} \xi}$ instead of infinity, where $\xi=-\Phi(r) / \sigma^{2}$. A calculation reveals that

$$
\eta=\frac{\left(1-\frac{\xi}{e^{\xi}-1}\right)\left(\frac{\sqrt{\pi}}{2} e^{\xi} \operatorname{Erf}(\sqrt{\xi})-\xi^{1 / 2}-\frac{2}{3} \xi^{3 / 2}\right)}{\frac{\sqrt{\pi}}{2} e^{\xi} \operatorname{Erf}(\sqrt{\xi})-\xi^{1 / 2}-\frac{2}{3} \xi^{3 / 2}-\frac{4}{15} \xi^{5 / 2}}
$$

where $\operatorname{Erf}(z)$ is the error function defined as $\operatorname{Erf}(z)=$ $2 / \sqrt{\pi} \int_{0}^{z} e^{-t^{2}} d t$. For large values of $\xi$, 


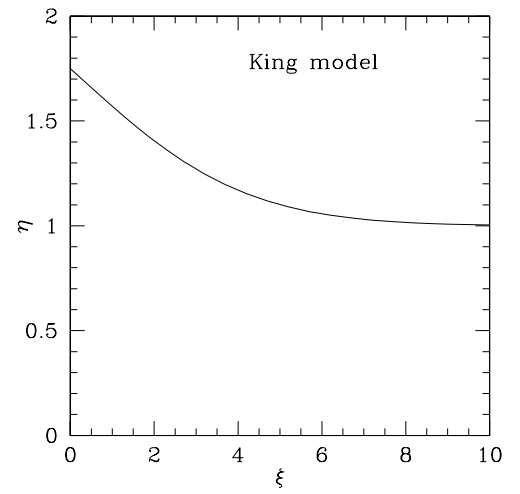

FIG. 1. A plot of $\eta$ as a function of $\xi=-\Phi(r) / \sigma^{2}$ for a King model. The Brownian particle at the center of a King stellar model approaches exact equipartition as $\xi$ becomes large.

$$
\eta \sim 1+\left(8 \xi^{3 / 2} / 15 \sqrt{\pi}-1\right) \xi e^{-\xi}+\cdots O\left(e^{-2 \xi}\right) \text { for } \xi \gg 1
$$

Fig. 1 shows the relation between $\eta$ and $\xi$. $\eta$ starts at a value of approximately 1.75 and falls asymptotically to 1 as $\xi$ rises. The King model goes over into the isothermal sphere as $\xi \rightarrow \infty$. The (mild) deviation from equipartition is because of the deviation of the distribution function from the Maxwellian form and, in particular, the existence of an upper velocity limit above which the distribution function vanishes. Actual stellar systems are well described by King models with the central potential chosen such that $-\Phi(0) / \sigma^{2}$ lies between roughly 3 and 10 ( [3], [6], [7], 8]). As Fig. 1 shows, black holes at the centers of such systems will be very close to equipartition with the stars.

Polytropic models. Consider next stellar systems described by a polytropic distribution function which takes the simple form

$$
f(E) \propto \begin{cases}(-E)^{n} & \text { if } E<0 \\ 0 & \text { if } E \geq 0 .\end{cases}
$$

Simple calculations give

$$
\eta=(n+5 / 2) /(n+1) \text { for } n>-1 .
$$

Note that $\eta$ for a polytropic model is independent of position $r$, unlike in the case of the King model. For large values of $n, \eta$ is close to 1 , but for small values of $n$, there can be significant deviations from equipartition.

The potential profile of a polytropic system is a solution of the Lane-Emden equation [3, which can be solved in terms of elementary functions in only two cases: $n=7 / 2$ (this case was considered in [4]) and $n=-1 / 2$. We examine these two cases in more detail.

Polytropic model with $n=7 / 2$. When $n=7 / 2$, we get the following functional forms for the density and potential profiles of the stellar system:

$$
\rho(r)=\frac{3 M a^{2}}{4 \pi} \frac{1}{\left(r^{2}+a^{2}\right)^{5 / 2}}, \quad \Phi(r)=\frac{-G M}{\left(r^{2}+a^{2}\right)^{1 / 2}},
$$

where $G$ is the gravitational constant, $M$ is the total mass of the stellar system in stars, and $a$ is a length parameter. This is the well-known Plummer model 99] which provides a good fit to some globular clusters. In this case, $\eta=4 / 3$, which is mildly different from equipartition. $\omega_{0}^{2}=k / m=$ $G M / a^{3}$ for small $r$, and

$$
\begin{gathered}
C=(8 G M / 9 a) \gamma m m_{\star}, \\
\left\langle x^{2}\right\rangle=2 a^{2} m_{\star} / 9 m, \quad\left\langle v_{x}^{2}\right\rangle=2 G M m_{\star} / 9 a m .
\end{gathered}
$$

Polytropic model with $n=-1 / 2$. When $n=-1 / 2$, we get the following functional forms for the density and potential profiles of the stellar system:

$$
\begin{gathered}
\rho(r)= \begin{cases}M /\left(4 \pi^{2} a^{2}\right) \times \sin (r / a) / r & \text { if } r<\pi a \\
0 & \text { if } r \geq \pi a,\end{cases} \\
\Phi(r)= \begin{cases}-G M \sin (r / a) / \pi r & \text { if } r<\pi a \\
-G M / r+G M / \pi a & \text { if } r \geq \pi a .\end{cases}
\end{gathered}
$$

In this case, $\eta=4$, which is very different from equipartition. $\omega_{0}^{2}=k / m=G M / 3 \pi a^{3}$ for small $r$, and

$$
\begin{gathered}
C=(8 G M / \pi a) \gamma m m_{\star}, \\
\left\langle x^{2}\right\rangle=6 a^{2} m_{\star} / m, \quad\left\langle v_{x}^{2}\right\rangle=2 G M m_{\star} / \pi a m .
\end{gathered}
$$

The expressions for $\left\langle x^{2}\right\rangle$ in Eqs. (22) and (24) agree with the result of Bahcall \& Wolf 10] to within factors of order unity.

Numerical simulations. In order to confirm our results, we have performed N-body simulations, using suitably modified versions of the fourth-order integrators of Aarseth [11] developed by Quinlan [12], for the two polytropic stellar systems described above. The techniques used are detailed in [4] and [12]. In both cases, we use units such that $G=M=1$, and Coulomb forces are softened by $\epsilon=5 \times 10^{-3}$ in order to prevent numerical divergences. Note that the polytropic initial conditions for the stellar system are not steady state solutions in the presence of the black hole; the initial transient response was therefore numerically settled.

For the case of the Plummer model, we have taken the mass of the black hole to be $m=0.01$, and the length scale of the potential to be $a=3 \pi / 16$. The stellar system is made up of 100,000 stars, each of mass $m_{\star}=1 \times 10^{-5}$. The model was integrated for 600 time units. The left half of Fig. 2 shows the binned distributions of one component of the black hole's position and velocity in the stationary state. The solid lines show the corresponding bin values as predicted by Eqs. (16) and (7), under the assumption that in the stationary state, ensemble averages will be equal to time averages over long time spans. The agreement with the predictions of the model is evidently good (these results were described in [4]). 

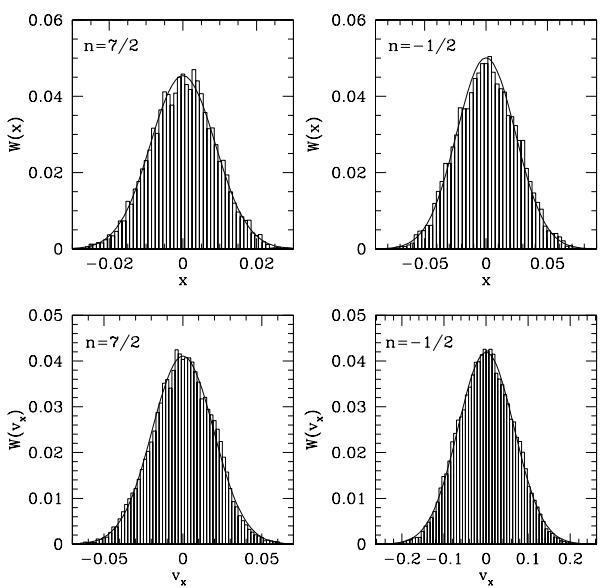

FIG. 2. The panels on the left show the distributions of $x$ and $v_{x}$ from simulation (bins) and theory (line) for a black hole of mass $m=0.01$ in a Plummer $(n=7 / 2$ polytrope) model made up of 100,000 stars of total mass $M=1$. The panels on the right show the corresponding results for a black hole of mass $m=0.03$ in a $n=-1 / 2$ polytrope model of total mass $M=1$ and 20,000 stars.

The right half of Fig. 2 shows the corresponding plots for the $n=-1 / 2$ polytrope. We have here taken $m=3 \times 10^{-2}$, and $a=3 / 4 \pi$. The stellar system is made up of 20,000 stars, each of mass $m_{\star}=5 \times 10^{-5}$. The integration time was 900 units. Agreement with the analytical results is again good. We note that the agreement seen in Fig. 2 would be spoiled if the appropriate value for $\eta$ were different from that given by our model.

Summary. We conclude that the model outlined in this Letter provides a good statistical description of the dynamics of a Brownian particle - such as a massive black hole - in a stellar system. If the black hole is inserted at the center of such a system, the transient effects of its initial position and velocity decay exponentially fast, and it settles into a stationary state in which the distributions of its position and velocity become time-independent. These distributions are also independent of each other and are Gaussian. If the stellar distribution function is Maxwellian, there is in the stationary state precise equipartition of kinetic energy between the black hole and the stars. The greater the deviation of the distribution function from a Maxwellian form, the greater is the deviation from strict equipartition. We have shown several examples of such cases, in one of which the black hole is very far from equipartition. The deviation originates from the existence of an upper velocity limit for stars beyond which the distribution function is zero. Note that the stationary state above is not a true equilibrium, since at very long time-scales (approximately 20 times the relaxation time), the stellar system will undergo core collapse [3].

The above analytical model applies strictly only to stellar models in which the density and potential profiles are non-singular at small radii; in realistic systems, however, the massive black hole would induce a density cusp consisting of stars bound tightly to it. Since it carries the cusp with it as it moves around, it is as if a black hole of a somewhat larger effective mass were moving in a background of unbound stars whose density profile is flat near the center (and unaffected by the black hole away from the center provided its mass is much smaller than the total mass of the stellar system). Since the restoring force and dynamical friction are provided mainly by the unbound stars, we would expect our results to apply to real systems as long as the mass of stars in the cusp is much smaller than the mass of the black hole; our simulations show that this is indeed so for the cases considered here.

Acknowledgments. We thank G. Quinlan for providing the simulation code. This work was supported in part by NASA grants NAG 5-7039, 5-7768, and by NSF grants AST-9900877, AST-0071019 (for AL).

[1] S. Chandrasekhar, Rev. Mod. Phys. 15, 1 (1943).

[2] S. Chandrasekhar, Astrophys. J. 97, 255 (1943).

[3] J. Binney \& S. Tremaine Galactic Dynamics (Princeton: Princeton University Press, 1987).

[4] P. Chatterjee, L. Hernquist \& A. Loeb, in press, Astrophys. J. (2002). (http://xxx.lanl.gov/abs/astroph/0107287)

[5] I. R. King, Astron. J. 71, 64 (1966).

[6] S. G. Djorgovski \& G. Meylan, Astron. J. 108, 1292 (1994).

[7] J. Kormendy, Astrophys. J. 218, 333 (1978).

[8] I. R. King, Astrophys. J. 222, 1 (1978).

[9] H. C. Plummer, Mon. Not. Roy. Astron. Soc. 71, 460 (1911).

[10] J. N. Bahcall \& R. A. Wolf, Astrophys. J. 209, 214 (1976).

[11] S. J. Aarseth, in Galactic Dynamics and N-Body Simulations, Ed. G. Contopoulos, N. K. Spyrou \& L. Vlahos (Springer-Verlag: Berlin, 1994).

[12] G. D. Quinlan \& L. Hernquist, New Astron. 2, 533 (1997). 